\title{
Biological network-inspired interpretable variational autoencoder
}

\author{
Lucas Seninge ${ }^{1}$, loannis Anastopoulos ${ }^{1}$, Hongxu Ding ${ }^{1, *} \&$ Joshua M Stuart ${ }^{1, *}$ \\ ${ }^{1}$ Department of Biomolecular Engineering and Genomics Institute, University of California, Santa Cruz, \\ Santa Cruz, California, USA \\ * Correspondence should be addressed to H.D (hding16@ucsc.edu) or J.S. (jstuart@ucsc.edu)
}

\begin{abstract}
Deep learning architectures such as variational autoencoders have revolutionized the analysis of transcriptomics data. However, the latent space of these variational autoencoders offers little to no interpretability. To provide further biological insights, we introduce a novel sparse Variational Autoencoder architecture, VEGA (Vae Enhanced by Gene Annotations), whose decoder wiring is inspired by a priori characterized biological abstractions, providing direct interpretability to the latent variables. We demonstrate the interpretability and flexibility of VEGA in diverse biological contexts, by integrating various sources of biological abstractions such as pathways, gene regulatory networks and cell type identities in the latent space of our model. We show that our model could recapitulate the mechanism of cellular-specific response to treatments, the status of master regulators as well as jointly investigate the cell type and cellular state identity in developing cells. We envision the approach could serve as an explanatory biological model in contexts such as development and drug treatment experiments.
\end{abstract}

\section{INTRODUCTION}

Recent advances in single-cell RNA sequencing (scRNA-Seq) technologies have enabled the characterization of cellular states at an unprecedented scale and resolution ${ }^{1}$. Among the many widely-used frameworks for analyzing complex transcriptomic patterns in single cells, Artificial Neural Networks (ANNs) such as Autoencoders (AEs) ${ }^{2}$ have emerged as powerful tools. AEs are neural networks aiming at transforming an input dataset into a decoded representation while minimizing the information loss ${ }^{3}$. The diversity in their architecture design makes AEs suitable to tackle various important challenges of scRNA-Seq analysis, such as dimensionality reduction ${ }^{4}$, clustering $^{5}$ and data imputation ${ }^{6}$.

More recently, deep generative models such as Variational Autoencoders ${ }^{7}$ (VAEs) have proven to be extremely useful for the probabilistic modeling of single-cell transcriptomes, such scVI or scGen ${ }^{8-10}$. While deep generative models have shown impressive performance for their dedicated modeling tasks, they often lack interpretability thus cannot offer a biologically meaningful latent representation of transcriptomes. For example, latent perturbation vectors extracted with scGen cannot be directly related to gene module variations ${ }^{10}$. 
Here we propose VEGA (Vae Enhanced by Gene Annotations), a Variational Autoencoder with a sparse linear decoder informed by biological networks. This model aims at offering an interpretable latent space to represent various biological information, e.g. the status of biological pathways or activity of transcriptional regulators. Specifically, the scope of our model is twofold, (1) encoding data over an interpretable latent space and (2) inferring biological information for out-of-sample data.

\section{RESULTS}

\section{Architectural design of VEGA}

To create a readily interpretable $V A E$, here we propose a novel architecture inspired by biological abstractions, as shown in Figure 1A. In many standard VAE implementations, the information bottleneck of the encoder-decoder architecture often represents latent variables modeled as a multivariate normal distribution. Despite providing highly informative representations of the input data, VAE latent variables are in general hard to interpret. To interpret $\mathrm{VAE}$ latent variables, Svensson et al. proposed to investigate the corresponding weights towards original variables, similarly to the interpretability offered by standard factor models such as $\mathrm{PCA}^{11}$. Although providing valuable insights in interpreting single cell biology, such an approach requires further statistical analyses on the weights of the decoder. The generative part of our model (decoder) directly relates the latent variables (biological abstractions, such as gene-regulatory networks) and the original gene features: a dependency between gene $k$ and latent variable $z^{(l)}$ exists if and only if gene $k$ is a priori annotated as part of biological abstraction $I$. This relies on the fundamental assumption that a set of known biological modules underlies and orchestrates the gene expression patterns of a given scRNA-Seq dataset. The annotation can come from curated databases such as MSigDB ${ }^{12}$, inferred gene-regulatory networks ${ }^{13}$, or any other pre-defined gene set. Such a design presents two main advantages: (1) the latent variables are initialized a priori therefore directly interpretable as the activity of biological modules, and (2) the flexibility in the biological module specification makes it generalizable to different biological abstractions (such as pathways, gene-regulatory network or even cell types, METHODS).

When initializing the architecture of such a model, it is possible to model the dependency between biological modules to the original features in the inference network (encoder), the generative network (decoder), or both. Our choice of using a deep encoder, sparse linear decoder structure can be justified by the goal of increasing the inference capacity of our model: it can capture more complex patterns in its interpretable latent space when approximating the true posterior distribution $P\left(z_{i} \mid x_{i}\right)$ over biological modules, while the link between latent variables and original gene features is guaranteed by the sparse linear decoder part of the neural network (Supplementary Figure 1).

\section{Recapitulating biological information over an interpretable latent space}


To demonstrate the ability of our proposed model to recapitulate the status of biological pathways, we applied it to a published Peripheral Blood Mononuclear Cells (PBMCs) dataset stimulated with the chemokine interferon- $\beta^{14}$ (METHODS). We first show that our model is able to capture cell types and stimulation status using the reactome pathways as latent variables (Fig.1B). We further investigated the activation status of individual pathways, specifically we show that the interferon- $\alpha / \beta$ signaling activity segregates stimulated and naive cells, confirming the ability of our model to capture pathway activity in its latent space (Fig.1C, Fig.1D). We further examined other known biological pathways involved in interferon-induced immune cell activation and found cell type-specific activation of certain cellular processes. For example, the tryptophan catabolism response to interferon separates innate immune cells (Dendritic cells, FCGR3A+Monocytes, CD14+Monocytes) from adaptive immune cells (NK cells, T-cell CD8, T-cell CD4, B cells) (Fig.1D), as previously investigated ${ }^{15,16}$. Together, these results show the ability of the model to project cells in an interpretable space, allowing investigation of cell-type specific patterns at the cellular process level.

It is often the case that the expected response to a stimulus is not known a priori. Differential gene expression has become a standard tool to study the difference in gene expression patterns between groups of samples. This procedure is also possible in deep generative networks modelling gene expression ${ }^{9}$. We propose a similar procedure to study the difference in biological module activity based on the latent space of our model: we can formulate alternative hypotheses and compute the ratio of their posterior probabilities using the variational posterior distribution of our model, which corresponds to the Bayes Factor (BF, METHODS). When applied to innate immune cells in the stimulated vs. control groups, we show that pathways that are expected to be activated in the stimulated groups (interferon signaling, tryptophan catabolism) are found to be significant by our method ( $\left|\log _{\mathrm{e}}(\mathrm{BF})\right|>3$, Fig.1E). We compared the results of our differential factor activity method with the False Discovery Rate (FDR) values of the standard GSEA toolkit (METHODS, Fig.1F). While both methods found the expected activation of the interferon- $\alpha / \beta$ signaling pathway in the stimulated groups, GSEA missed the tryptophan catabolism activation in innate immune cells (Fig.1F). Overall, VEGA does not seem to suffer as much from the gene set size bias problem inherent to GSEA (Fig.1F, Supplementary Figure 2), suggesting more context-specific significant hits given the biology of the dataset.

\section{Large-scale investigation of biological responses to drug treatments in cell lines}

Next, we investigated whether our model could capture and recapitulate patterns of drug responses in large-scale experiments over cancer cell lines, such as introduced in recent experimental protocols like MIX-Seq ${ }^{17}$. To this end, we gathered single-cell data for 97 cancer cell lines under 5 different conditions: 24 hour DMSO treatment (control), 24 hour Trametinib treatment (MEK inhibitor), 24 hour Dabrafenib treatment (Mutated BRAF inhibitor), 24 hour Navitoclax treatment (Bcl-2 inhibitor) and 24 hour BRD3379 treatment (tool compound with unknown mode of action, MoA) (METHODS). We trained one model for each different drug treatment (4 models in total) by combining the drug treatment dataset and the control group (DMSO dataset), using the hallmark gene sets from $\mathrm{MSigDB}^{18}$ to initialize the latent space. Overall, each model was able to recapitulate the biological response of cell lines to each 
treatment (Fig.2A, Supplementary Figure 3). For Trametinib notably, the important change in G2M checkpoint activity (decrease in the treated condition) agrees with the expected MoA of a MEK inhibitor ${ }^{19,20}$ (Fig.2B). Next, we sought to investigate whether we could recapitulate the pattern of biological responses between control and treated conditions for each cell line/drug treatment pair. For each pair, we computed the Bayes Factor of each latent variable (pathway) in our models. The resulting heatmap can be used to understand and interpret patterns of response over all experimental conditions (Fig.2C). As found when investigating the low-dimensional embedding of each dataset, Trametinib resulted in the strongest transcriptional response of all studied drugs. Notably, the Trametinib-specific interferon- $\alpha$ and interferon- $\gamma$ response was correctly recapitulated by our model, as previously shown both experimentally ${ }^{21}$ and by the original MIX-Seq authors ${ }^{17}$. Furthermore, we found that Dabrafenib treated BRAF-mutant melanoma cell lines exhibited a strong transcriptional response, clustering with the Trametinib cell lines as reported in the MIX-Seq study (Fig.2.C, Supplementary Figure 3). Overall, the results presented here agree with previous gene set analysis results on this dataset, and demonstrate the ability of our model to recapitulate patterns of drug response in large-scale experiments.

\section{Gene regulatory analysis of glioblastoma reveals stratification of neoplastic cells} As previously mentioned, one of the major strengths of our model is the flexibility in the latent space specification, as any biological module can be related to a gene set and therefore be encoded in our model. One of the most important aspects of biology is the tight regulation of gene expression by transcription factors ${ }^{22}$. Analyzing the activity of those master regulators is important in understanding biological states like cell types or diseases, as dysregulation in their activity can have dramatic impact on gene expression programs and phenotypes ${ }^{23,24}$. To this end, we investigated whether using master regulators to specify the latent space of our model could help understand the underlying gene regulatory networks (GRN) in the context of a single-cell glioblastoma (GBM) dataset ${ }^{25}$. We used the GBM ARACNe ${ }^{13}$ network reported in ${ }^{24}$ to guide the structural design of our model. Specifically, the transcription factors were used to initialize the latent space of our model, and the transcriptional factor-target connections were used initializing the decoder wiring. After training the model, we show that pre-annotated cell types can be recapitulated in the latent space (Fig.2D). We examined the activity of STAT3 and OLIG2, two well-known master regulators of the mesenchymal (MES) and proneural (PN) GBM subtypes, respectively. We confirmed that their activity inferred in the latent space was anticorrelated in neoplastic cells (Fig.2E). Additionally, OLIG2, as a known master regulator of oligodendrocytes differentiation ${ }^{26}$, was inferred as activated in Oligodendrocyte precursor cells (OPCs). These results suggest the flexibility and extensibility of our method in the specification of the latent space and decoder wiring. Furthermore, these results demonstrate the ability of our model to faithfully represent biological information.

\section{Combining cell type and cellular state representations refines cortical organoid development analysis}


A great challenge of modern cellular biology is to identify and define cell types and cellular states in order to systematically study homeostasis and disease development under a common vocabulary. To this end, we investigated whether we could combine information about cell types (in the form of marker sets defined as lists of marker genes) and cell states (reactome pathways) in the latent space of our model, to produce a disentangled representation of cell types and cellular states. We applied our model to a dataset of cells during the early development of cortical organoids from Field et al. ${ }^{27}$, including the information about the major cell types defined in the study in the latent space (Fig.3A). After training, the activity of each cell type marker set was able to correctly segregate its corresponding cell type as annotated by the original authors (Fig.3B-D). Moreover, in a "one vs. rest" differential factor analysis setting for each cell type population, the activity of the corresponding marker set showed a significant enrichment $\left(\left|\log _{e}(B F)\right|>3\right)$, potentially showing the opportunity for researchers to use the latent activity of cell type marker sets to annotate unknown clusters (Fig.3E). To study whether our model could separate cell type identity from cellular states such as dividing versus quiescent cell populations, we projected the dataset into two components: (1) the neural epithelium marker set activity (a type of early brain progenitor) and (2) the cell cycle mitotic pathway activity (Fig.3F). As discussed previously, the neural epithelium marker set activity separates the neural epithelium cells from the rest of the dataset, while the activity of the cell cycle mitotic pathway separated quiescent from actively dividing cells in the two progenitors populations (radial glia cells and neural epithelium). To validate that the cells identified as dividing were proliferating, we studied the correlation between the cell cycle mitotic pathway activity and the expression of the MKI67 gene, a canonical marker of proliferation (external validator not present in the cell cycle mitotic pathway set) (Fig.3G). Overall, the expression of MKI67 correlates well with the inferred activity of the cell cycle mitotic pathway $\left(R^{2}: 0.67\right)$. Together, these results demonstrate the potential use of our model to jointly infer cell type and cellular state identity for different populations of cells, as combining different sources of information (pathways, master regulators, cell type markers) in the latent space of our model can shed light on different aspects of the identity of a single-cell.

\section{Generalization of the inference process to out-of-sample data}

We posit that our model can be used to infer an interpretable latent representation of data unseen at the time of training, which we refer to as out-of-sample data. To confirm the generalizability, we evaluate our model in two settings: (1) the "biological generalization" of the inference, where a certain cell type/condition pair is left out during training on the Kang et al. PBMC dataset in a similar way to scGen ${ }^{10}$ (intra-dataset setting), and (2) the "technical generalization" of the inference, where a model trained on the Kang et al. ${ }^{14}$ PBMC dataset (which we refer to as study $A$ ) is evaluated on another PBMC dataset containing only control cells from Zheng et al. ${ }^{28}$ (study B, inter-dataset setting).

For the "biological generalization", we first checked that the distribution of the interferon- $\alpha / \beta$ signaling pathway activity in the out-of-sample stimulated CD4 T-cells matched the inferred activity in the in-sample CD4 T-cells (Fig.4A). To perform a more systematic comparison of the inferred latent space between out-of-sample and in-sample cells, we used our Bayesian Differential Factor activity procedure (METHODS) between (1) stimulated in-sample cells and 
control cells for a given cell type (model trained with the whole dataset) and (2) stimulated out-of-sample cells and control cells for the same cell type (model trained with one cell type/condition pair left out), and checked the amount of overlaps in the top 50 differentially activated factors (Fig.4B). The results suggested consistency between the in-sample and out-of-sample differentially activated factors, with an average $72 \%$ overlaps. To further evaluate the capacity of data reconstruction, we measured the $\mathrm{R}^{2}$ between the original and decoded data in the in-sample and out-of-sample settings (Fig.4C). We show that the $\mathrm{R}^{2}$ decreases only marginally in the out-of-sample setting, confirming the ability of the model to generalize to unseen data produced in a similar experimental setting.

For the "technical generalization", we again checked that the interferon- $\alpha / \beta$ pathway activity distribution of study B encoded control CD4 T-cells matched that of study A control CD4 T-cells (Fig.4D). We also investigated whether the top 50 differential factors of each cell type in a "one-vs-rest" differential setting for the control cells of study A overlapped with a similar procedure performed on the control cells of study B (Fig.4E). We show that on average $67 \%$ of the top 50 differential factors for study A overlap with those of study $B$, showing that the model can generalize across studies unseen at the time of training. We then study whether the model can use the inferred latent space to accurately reconstruct the original expression profiles of both studies. We show that the $R^{2}$ between original and reconstructed cells of study $B$, although lower than those for study $A$, improves upon the baseline correlation between the expression profiles of study A vs study B for most of the cell types (Fig.4F).

We believe that these results together show the ability of the model to infer an interpretable latent space for data unseen at the time of training, with some limitations coming from potential batch effects. This could be easily alleviated by re-training the model on the new data, as this procedure is quite fast.

\section{DISCUSSION}

In this study, we introduced a novel VAE architecture, VEGA, whose decoder wiring is inspired by known biology to infer the activity of various biological modules at the single-cell level. By encoding single-cell transcriptomics data into an interpretable latent space specified a priori, our method provides a fast and efficient way of analyzing the activity of various biological abstractions in different contexts. We demonstrated its use in understanding the response of specific cell type populations to different perturbations, providing interpretable insights on biological module activity. We envision that our model could be useful to prioritize drugs based on pathway expression in cancer, as studying the response of specific cell populations can be useful to understand drug sensitivity and resistance. Integrating drug response prediction models with explanatory models like ours could be highly beneficial in designing novel therapeutic strategies. Furthermore, we also speculate that our interpretable VAE will provide insights in understanding general biological questions.

The flexibility in the specification of the latent space paves the way for analyzing the activity of biological modules such as pathways, transcriptional regulators and even cell type-specific 
modules as demonstrated in this study. We further showed that our model could be used to explore together the cell type and cell state of various subpopulations of cells, whether it is in the context of perturbation experiments or not. Additionally, the weights of decoder connections provide direct interpretability of the relationship between the latent variables and the original features. For example, it could be used to contrast interaction confidence in inferred gene-regulatory networks, or rank genes by their importance in a certain biological module in a data-driven way.

The clear limitations of the current architecture resides in the sparse, single-layer decoder of the model. In fact, such an architectural design prevents the further improvement of generalizability and robustness. As a consequence, the generative capacity of our VAE is undermined. For example, while theoretically our model could be used for interpretable response prediction using latent vector arithmetics in a similar fashion to $\mathrm{scGen}^{10}$, the limited generative capacity of our approach sacrifices predictive performance for interpretability. Our design choices therefore compromise those aspects for biological interpretability of the latent space. We believe advanced insights in network biology, e.g. multi-layer GRNs that can describe regulatory machinery more comprehensively, could alleviate these limitations. This would open the possibility to perform targeted, in-silico activation and repression of biological programs on specific cell populations to study its effect on development or disease progression.

On the other hand, hard-coded connections of the linear decoder does not leave any room for correcting prior knowledge about biological factors when the context requires it, as is the case in other latent variable models such as $\mathrm{f}_{-} \mathrm{sLLVM}^{29}$. In fact, prior biological knowledge obtained from existing databases like MSigDB can be incomplete or not context-specific, as additional unannotated genes can play an important role in certain biological factors. We believe that this could be easily improved by using standard regularization methods on the weights of the decoder instead of masking. We leave this to further explorations.

\section{METHODS}

\section{The VEGA architecture}

We introduce here the architecture of a Variational Autoencoder (VAE) for biological modules analysis. Our VAE is a deep generative model that aims at maximizing the likelihood of a single-cell dataset $X$ under a generative process ${ }^{7,10}$ described as:

$$
P(X \mid \theta)=\int P(X \mid Z, \theta) P(Z \mid \theta) d Z
$$

With $\theta$ being the learnable parameters of a neural network. Here, we choose to have our set of latent variables $Z$ explicitly represent biological abstractions, such as pathways, gene regulatory 
networks or cell type marker sets. To enforce this, we modify the decoder part of the VAE architecture to be a single layer, masked, linear decoder. Specifically, the connection of this layer, between latent node $z^{(j)}$ and input gene features, are specified using a mask $M$, which is a binary matrix where:$$
M_{i, j}= \begin{cases}1 & \text { if gene } i \text { is a member of gene set } j, \\ 0 & \text { otherwise }\end{cases}
$$

During training, we zero-out gradients associated with masked weights, such that backpropagation only applies to weights originating from a priori annotation, while the rests of the weights are kept at 0 . Additionally, the decoder is constrained to positive weights $(w \geq 0)$, to maintain interpretability as to the directionality of the biological module activity. Having explicitly specified the connections between genes and latent variables in the generative part of our model, we ensure that the latent space represents biological module activity, as well as its interpretability. We choose to model the set of latent variable $Z$ as a multivariate normal distribution, parametrized by our inference network with learnable parameters $\phi$ as such:

$$
Q(Z \mid X, \phi)=\mathcal{N}\left(\mu_{\phi}(X), \Sigma_{\phi}(X)\right)
$$

This choice of variational distribution is common and has proven to work well in previous single-cell studies ${ }^{9,10}$. The objective to be maximized when training our model is the Evidence of Lower Bound (ELBO), similarly to many standard VAE implementations ${ }^{7,10}$ :

$$
\mathcal{L}(X)=\mathbb{E}_{Q(Z \mid X, \phi)}[\log P(X \mid Z, \theta)]-K L(Q(Z \mid X, \phi) \| P(Z \mid \theta))
$$

, where the expectation over the variational distribution can be approximated using Monte Carlo integration over a minibatch of data, and the Kullblack-Leibler divergence term has a closed-form solution as we set the prior $P(Z \mid \theta) \sim \mathcal{N}(0, \mathbf{I})$. The reparametrization $\operatorname{trick}^{7}$ is used when sampling our variational distribution to allow standard backpropagation to be applied when training the model.

To retain information of genes that are not present in our pre-annotated biological networks, we add additional fully-connected nodes to the latent space of our model. This has two effects: (1) it allows to model the expression of unannotated genes, crucial for a good reconstruction of the data during training, and (2) it can help capture additional variance of the data that is unexplained by the existing biological factors, considerably improving the training of the model.

\section{Bayesian differential factor activity}

It can be of major interest to study the difference in factor activation between two groups of cells and its significance. To this end, we draw inspiration from the Bayesian differential gene 
expression procedure introduced in ${ }^{9}$ and propose a similar differential factor analysis procedure. For a given factor $k$, a pair of cells $\left(x_{a}, x_{b}\right)$ and their respective group ID $\left(s_{a}, s_{b}\right)$ (e.g two different treatment conditions), our two mutually exclusive hypotheses are:

$$
\mathcal{H}_{0}^{k}:=\mathbb{E}_{s}\left[z_{a}^{k}\right]>\mathbb{E}_{s}\left[z_{b}^{k}\right] \text { versus } \mathcal{H}_{1}^{k}:=\mathbb{E}_{s}\left[z_{a}^{k}\right] \leq \mathbb{E}_{s}\left[z_{b}^{k}\right]
$$

This can intuitively be seen as testing whether a cell has a higher mean biological factor activation than another, the expectation representing empirical frequency. We evaluate the most probable hypothesis by studying the log-Bayes factor $K$ defined as:

$$
K=\log _{e} \frac{p\left(\mathcal{H}_{0}^{k} \mid x_{a}, x_{b}\right)}{p\left(\mathcal{H}_{1}^{k} \mid x_{a}, x_{b}\right)}
$$

Here, the sign of $K$ tells us which hypothesis is more likely, and the magnitude of $K$ encodes a significance level. Having access to the conditional posterior distribution $q(Z \mid X)$ over the factor activation (the inference part of our model), we can approximate each hypothesis probability distribution as:

$$
p\left(\mathcal{H}_{0}^{k} \mid x_{a}, x_{b}\right) \approx \sum_{s} p(s) \underset{\sup .\left(z_{a}\right), \sup .\left(z_{b}\right)}{\iint_{a}} p\left(z_{a}^{k}>z_{b}^{k}\right) d q\left(z_{a}^{k} \mid x_{a}\right) d q\left(z_{b}^{k} \mid x_{b}\right)
$$

where $p(s)$ is the relative abundance of cells in group $s$, and the integrals are approximated with direct Monte Carlo sampling.

Similarly to ${ }^{9}$, assuming cells are independent, we can compute the average Bayes factor across many cell pairs randomly sampled from each group respectively. This helps us decide whether a factor is activated at a higher frequency in one group or the other. Through the paper, we consider factors to be significantly differentially activated if the absolute value of $K$ is greater than 3 (equivalent to an odds ratio of $\approx 20$ ).

\section{Datasets and preprocessing}

\section{Kang et al. dataset}

The Kang et al. ${ }^{14}$ dataset consisted of two groups of PBMCs, one control and one stimulated with interferon- $\beta$. We chose to use the same preprocessing steps as described by scGen authors ${ }^{10}$. Briefly, cells were annotated using the maximum correlation to one of the eight original cell type clusters identified, using an average of the top 20 cluster genes. Then data were filtered to remove cells with less than 500 genes expressed and genes expressed in 5 or less cells. Count per cells were then normalized and log-transformed, and we selected the top 
6998 highly variable genes, resulting in a final dataset of 18,868 cells. Raw data is available at GSE96583.

\section{Zheng et al. dataset}

The Zheng et al. ${ }^{28}$ dataset consists of $3 \mathrm{~K}$ PBMCs from an healthy donor. After filtering the cells, the count per cells were normalized and log-transformed. We then subset the genes to use the same 6998 genes of the Kang et al. PBMC dataset. The final dataset has 2623 cells and 6998 genes. Raw data are available at

https://support.10xgenomics.com/single-cell-gene-expression/datasets/1.1.0/pbmc3k.

\section{MIX-Seq dataset}

The MIX-Seq ${ }^{17}$ datasets were obtained from https://figshare.com/s/139f64b495dea9d88c70, and we used the data from experiment 3 to have enough cells to carry a smooth training of our model. For the 5 available datasets (97 cell lines treated with respectively DMSO, Trametinib, Dabrafenib, Navitoclax and BRD3379), we removed cells with 200 or less expressed genes, and genes expressed in less than 3 cells. We then normalized the number of counts per cell, and log-transformed the data. Finally, each dataset that was a drug treatment experiment was combined with a copy of the control dataset (DMSO treatment), and we extracted the top 5000 highly variable genes. This resulted in final datasets of size (16732 cells, 4999 genes) for the Trametinib+DMSO data, (16942 cells, 5000 genes) for the Dabrafenib+DMSO data, (14507 cells, 5000 genes) for the Navitoclax+DMSO data, and (15304 cells, 5000 genes) for the BRD3379+DMSO data.

\section{Darmanis et al. dataset}

The raw glioblastoma data from Darmanis et al. ${ }^{25}$ were obtained from http://www.gbmseq.org/ and preprocessed as followed: we removed cells with 200 or less expressed genes, and genes expressed in 3 or less cells. Count per cells were normalized and data were then log-transformed. Finally, we restricted the transcriptome to the top 6999 highly variable genes. The final dataset had a total of 3566 cells. Raw data is available at GSE84465.

\section{Field et al. dataset}

The cortical organoid data from Field et al. ${ }^{27}$ was processed similarly to the glioblastoma dataset. After normalization and highly variable genes selection, the dataset had a total of 4378 cells, with 6999 genes. Raw data is available GSE106245.

\section{Choice of gene annotations for the latent space of VEGA}

When initializing the latent space of our model, we chose to use pre-annotated gene sets from the Molecular Signature Database $(\mathrm{MSigDB})^{12}$. In particular, we chose to use the hallmark gene sets annotation (50 gene sets) or the Reactome database (674 gene sets). Reactome was used for the stimulated PBMCs analysis, and hallmark $\mathrm{H}$ was used in the MIX-Seq analysis part of this study. 
For the gene regulatory network analysis of glioblastoma cells, we derived an ARACNe ${ }^{13,30}$ network from bulk RNA-Seq samples of glioblastoma. Specifically, this network was obtained from a previously published paper ${ }^{31}$ and repurposed for the study of glioblastoma single-cell transcriptomics profiles.

For the cell type marker genes in the cortical organoid analysis, we contacted the authors to obtain the most relevant genes used in annotating those cell types. The marker list is available as a supplementary table.

\section{Dimensionality reduction for visualization}

For visualizing datasets, we used the UMAP algorithm ${ }^{32}$ as implemented in the umap python package. We used default parameters except for the min_dist parameter that we set to 0.5 . We also used t-SNE ${ }^{33}$ as implemented in the sklearn python package, with default parameters.

\section{Comparison with GSEA}

We ran Gene Set Enrichment Analysis ${ }^{12}$ (GSEA) using the prerank function from the gseapy package in Python. Briefly, we calculated differential expression scores for each gene between the control and treatment group using a Wilcoxon rank-sum test, as implemented in the rank_genes_groups functionality of the Scanpy package ${ }^{34}$. We ranked genes according to their test statistics, and ran GSEA prerank with the following settings: a minimum gene set size of 5 , a maximum gene set size of 1000 , and 1000 permutations. We ranked gene sets according to their FDR and considered significant hits when FDR $\leq 0.05$. When the FDR returned by GSEA was equal to 0 , we replaced it with $1 \mathrm{e}-5$ (to avoid math error when taking the logarithm).

\section{Evaluation metrics}

Silhouette scores were calculated to evaluate biological separation in the latent space of our model. We used euclidean distance in the latent space to compute silhouette coefficient of each cell $i$ defined as:

$$
s(i)=\frac{b(i)-a(i)}{\max \{a(i), b(i)\}}
$$

where $a(i)$ and $b(i)$ are respectively the mean intra-cluster distance and the mean nearest-cluster distance for cell $i$. We used either the stimulation or cell type labels from Kang et al. ${ }^{14}$ to assess the biological relevance of the latent space of our model (see Supplementary note 1). The sklearn package ${ }^{35}$ silhouette_score implementation was used for computation.

\section{ACKNOWLEDGMENTS}

The authors would like to thank Dr. David Haussler and Dr. Sofie Salama for their support. L.S would also like to thank David Parks for the useful feedback during the early development of the method. We also would like to thank Dr. Maximilian Haeussler for the feedback on the manuscript. 


\section{AUTHOR CONTRIBUTIONS}

L.S and I.A conceived the idea. L.S implemented the method and gathered the data. L.S and I.A performed the analysis. H.D and J.M.S supervised the research. All authors contributed to the manuscript.

\section{FIGURE LEGENDS}

Figure 1. Designing a novel VAE architecture with interpretable latent space. (A) Overview of the VEGA model. Composed of a deep non-linear encoder and a masked linear decoder, VEGA encodes single-cell transcriptomics data in an interpretable latent space specified a priori. (B) UMAP embedding of the latent space of VEGA retains the biological signal of the Kang et al. PBMCs dataset ${ }^{14}$. (C) Inferred interferon-alpha/beta signaling pathway activity segregates stimulated cells from the control population. (D) Bivariate factor plot showing the ability of the model to recover the tryptophan catabolism activity, an innate immune cell-specific response to the perturbation. (E) Volcano plot showing differentially active biological factors between stimulated and control innate immune cells. The red dots indicate factors with $\mid \log _{\mathrm{e}}$ (Bayes Factor)| $>3$ and a Mean Absolute Difference (MD) in the latent space of at least 5. (F) Comparison of the VAE Bayes Factor with GSEA - $\log _{10}(F D R)$. The size of the dots indicates the gene set size. The red, blue and purple quadrants correspond respectively to significant hits unique to our model, unique to GSEA, and common to both.

Figure 2. The flexibility in the latent space specification sheds light on the activity of various biological abstractions. (A) tSNE embedding of the latent space of our model for the MIX-Seq data ${ }^{17}$. The color indicates the treatment condition, and the arrow indicates the median shift in coordinates of each cell line between the 2 conditions. (B) Inferred G2M checkpoint activity of each cells, showing a decrease activity in the treated condition, as expected from the MoA of Trametinib. (C) Heatmap with hierarchical clustering showing the average $\log _{e}$ (Bayes Factor) of each pathway for each cell line/drug treatment pair (test between DMSO and treatment condition). Each row corresponds to a hallmark gene set, and each column to a different cell line/drug pair. First row of color indicates the drug, and the second row of color indicates the tissue identity (Tissue legend available in Supplementary Figure 2). Highlighted cell lines correspond to BRAF-mutant melanoma. Highlighted activities correspond to Trametinib-specific responses. (D) tSNE embedding of the latent space of the model for the glioblastoma dataset ${ }^{25}$, colored by cell type or $(E)$ Inferred activity of the master regulators STAT3 and OLIG2.

Figure 3. Disentangling cellular states and cell types in the early development of cortical organoids. (A) UMAP embedding of the latent space of our model for the week 2 cortical organoid dataset ${ }^{27}$. The cell type annotation corresponds to the original paper annotation. $(B, C, D)$ The inferred activity of each cell type factor (as defined by marker genes) correctly identifies the three main sub-populations of cells. (E) One-versus-rest differential factor analysis 
of each cell type population provides statistical significance for each cell type signature. Significance threshold for positive enrichment was set to $\log _{e}(B F)>3$. (F) Identification of dividing and quiescent sub-populations of neural progenitors using pathway and cell type activity projection. (G) CELL_CYCLE_MITOTIC pathway activity correctly identifies dividing cells as reported by its correlation with MKI67 gene expression (external canonical marker of dividing cells).

Figure 4. Generalization of the proposed VAE architecture to out-of-sample data. (A) Violin plot representing the distribution of the interferon- $\alpha / \beta$ pathway activity in control CD4-T cells, stimulated CD4 T-cells unseen at the time of training (out-of-sample) and stimulated CD4-T cells when included in the training procedure (in-sample). (B) Proportion of overlap in the top 50 differentially activated factors in the in-sample and out-of-sample settings with stimulated vs. control differential procedures for the seven main cell types in the study. Error bars correspond to standard deviation over 100 random sampling. (C) $R^{2}$ between the mean expression of real and reconstructed cells in the in-sample and out-of-sample settings for the seven main cell types of the study. Error bars correspond to standard deviation over 100 random sampling. (D) Violin plot of distribution of the interferon- $\alpha / \beta$ pathway activity in control CD4-T cells of study $A$ (Kang et al. ${ }^{14}$ ), stimulated CD4-T cells of study A and control CD4-T cells of study B (Zheng et al. ${ }^{28}$ ). (E) Proportion of overlap in the top 50 differentially activated factors of each study with one vs. rest differential procedures for the control cells of the seven main PBMC cell types. Error bars correspond to standard deviation over 100 random sampling. $(F) R^{2}$ between the mean expression of real and reconstructed cells of study A (Study A), mean expression of real and reconstructed cells of study $B$ (Study $B$ ) and mean expression of real cells of study $A$ and real cells of study B (Study A vs Study B). Error bars correspond to standard deviation over 100 random sampling.

\section{REFERENCES}

1. Kolodziejczyk, A. A., Kim, J. K., Svensson, V., Marioni, J. C. \& Teichmann, S. A. The technology and biology of single-cell RNA sequencing. Mol. Cell 58, 610-620 (2015).

2. Hinton, G. E. Reducing the Dimensionality of Data with Neural Networks. Science 313, 504-507 (2006).

3. Baldi, P. Autoencoders, Unsupervised Learning, and Deep Architectures. 14.

4. Wang, D. \& Gu, J. VASC: Dimension Reduction and Visualization of Single-cell RNA-seq Data by Deep Variational Autoencoder. Genomics Proteomics Bioinformatics 16, 320-331 (2018). 
5. Geddes, T. A. et al. Autoencoder-based cluster ensembles for single-cell RNA-seq data analysis. BMC Bioinformatics 20, 660 (2019).

6. Eraslan, G., Simon, L. M., Mircea, M., Mueller, N. S. \& Theis, F. J. Single-cell RNA-seq denoising using a deep count autoencoder. Nat. Commun. 10, 390 (2019).

7. Kingma, D. P. \& Welling, M. Auto-Encoding Variational Bayes. ArXiv13126114 Cs Stat (2014).

8. Way, G. P. \& Greene, C. S. Extracting a biologically relevant latent space from cancer transcriptomes with variational autoencoders. in Biocomputing 2018 80-91 (WORLD SCIENTIFIC, 2017). doi:10.1142/9789813235533_0008.

9. Lopez, R., Regier, J., Cole, M. B., Jordan, M. I. \& Yosef, N. Deep generative modeling for single-cell transcriptomics. Nat. Methods 15, 1053-1058 (2018).

10. Lotfollahi, M., Wolf, F. A. \& Theis, F. J. scGen predicts single-cell perturbation responses. Nat. Methods 16, 715-721 (2019).

11. Svensson, V., Gayoso, A., Yosef, N. \& Pachter, L. Interpretable factor models of single-cell RNA-seq via variational autoencoders. Bioinformatics 36, 3418-3421 (2020).

12. Subramanian, A. et al. Gene set enrichment analysis: A knowledge-based approach for interpreting genome-wide expression profiles. Proc. Natl. Acad. Sci. 102, 15545-15550 (2005).

13. Margolin, A. A. et al. ARACNE: An Algorithm for the Reconstruction of Gene Regulatory Networks in a Mammalian Cellular Context. BMC Bioinformatics 7, S7 (2006).

14. Kang, H. M. et al. Multiplexed droplet single-cell RNA-sequencing using natural genetic variation. Nat. Biotechnol. 36, 89-94 (2018).

15. Mellor, A. L., Lemos, H. \& Huang, L. Indoleamine 2,3-Dioxygenase and Tolerance: Where Are We Now? Front. Immunol. 8, (2017). 
16. Sorgdrager, F. J. H., Naudé, P. J. W., Kema, I. P., Nollen, E. A. \& Deyn, P. P. D.

Tryptophan Metabolism in Inflammaging: From Biomarker to Therapeutic Target. Front.

Immunol. 10, (2019).

17. McFarland, J. M. et al. Multiplexed single-cell transcriptional response profiling to define cancer vulnerabilities and therapeutic mechanism of action. Nat. Commun. 11, 4296 (2020).

18. Liberzon, A. et al. The Molecular Signatures Database Hallmark Gene Set Collection.

Cell Syst. 1, 417-425 (2015).

19. Kurata, K. et al. Growth arrest by activated BRAF and MEK inhibition in human anaplastic thyroid cancer cells. Int. J. Oncol. 49, 2303-2308 (2016).

20. Joshi, M., Rice, S. J., Liu, X., Miller, B. \& Belani, C. P. Trametinib with or without Vemurafenib in BRAF Mutated Non-Small Cell Lung Cancer. PLoS ONE 10, (2015).

21. Lulli, D., Carbone, M. L. \& Pastore, S. The MEK Inhibitors Trametinib and Cobimetinib Induce a Type I Interferon Response in Human Keratinocytes. Int. J. Mol. Sci. 18, (2017).

22. Spitz, F. \& Furlong, E. E. M. Transcription factors: from enhancer binding to developmental control. Nat. Rev. Genet. 13, 613-626 (2012).

23. Lee, T. I. \& Young, R. A. Transcriptional Regulation and Its Misregulation in Disease. Cell 152, 1237-1251 (2013).

24. Carro, M. S. et al. The transcriptional network for mesenchymal transformation of brain tumours. Nature 463, 318-325 (2010).

25. Darmanis, S. et al. Single-Cell RNA-Seq Analysis of Infiltrating Neoplastic Cells at the Migrating Front of Human Glioblastoma. Cell Rep. 21, 1399-1410 (2017).

26. Lu, Q. R. et al. Common Developmental Requirement for Olig Function Indicates a Motor Neuron/Oligodendrocyte Connection. Cell 109, 75-86 (2002).

27. Field, A. R. et al. Structurally Conserved Primate LncRNAs Are Transiently Expressed 
during Human Cortical Differentiation and Influence Cell-Type-Specific Genes. Stem Cell Rep. 12, 245-257 (2019).

28. Zheng, G. X. Y. et al. Massively parallel digital transcriptional profiling of single cells.

Nat. Commun. 8, 1-12 (2017).

29. Buettner, F., Pratanwanich, N., McCarthy, D. J., Marioni, J. C. \& Stegle, O. f-scLVM: scalable and versatile factor analysis for single-cell RNA-seq. Genome Biol. 18, 212 (2017).

30. Lachmann, A., Giorgi, F. M., Lopez, G. \& Califano, A. ARACNe-AP: gene network reverse engineering through adaptive partitioning inference of mutual information.

Bioinformatics 32, 2233-2235 (2016).

31. Ding, H. et al. Quantitative assessment of protein activity in orphan tissues and single cells using the metaVIPER algorithm. Nat. Commun. 9, 1471 (2018).

32. Mclnnes, L. \& Healy, J. UMAP: Uniform Manifold Approximation and Projection for Dimension Reduction. ArXiv180203426 Cs Stat (2018).

33. Maaten, L. van der \& Hinton, G. Visualizing Data using t-SNE. J. Mach. Learn. Res. 9, 2579-2605 (2008).

34. Wolf, F. A., Angerer, P. \& Theis, F. J. SCANPY: large-scale single-cell gene expression data analysis. Genome Biol. 19, 15 (2018).

35. Pedregosa, F. et al. Scikit-learn: Machine Learning in Python. J. Mach. Learn. Res. 12, 2825-2830 (2011). 


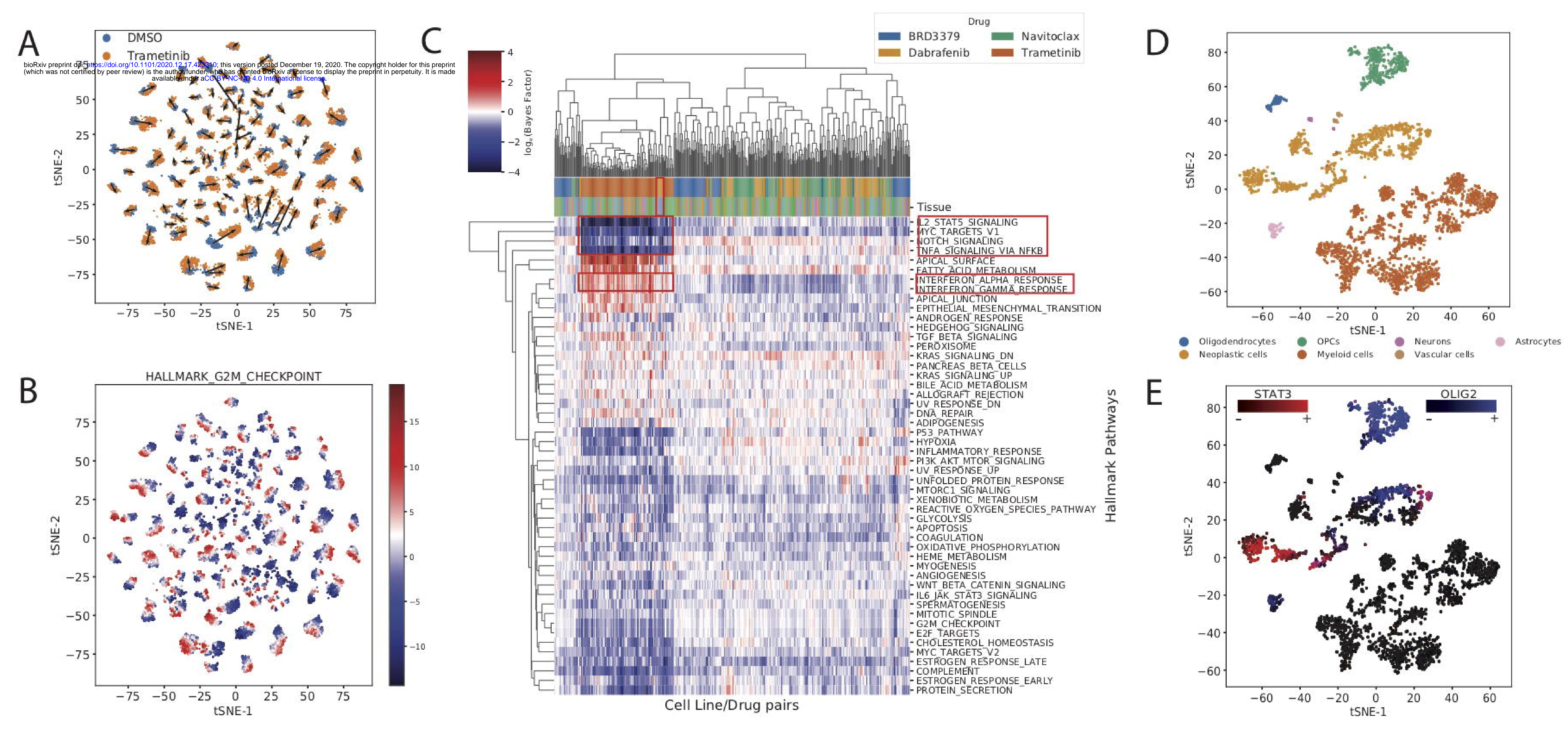



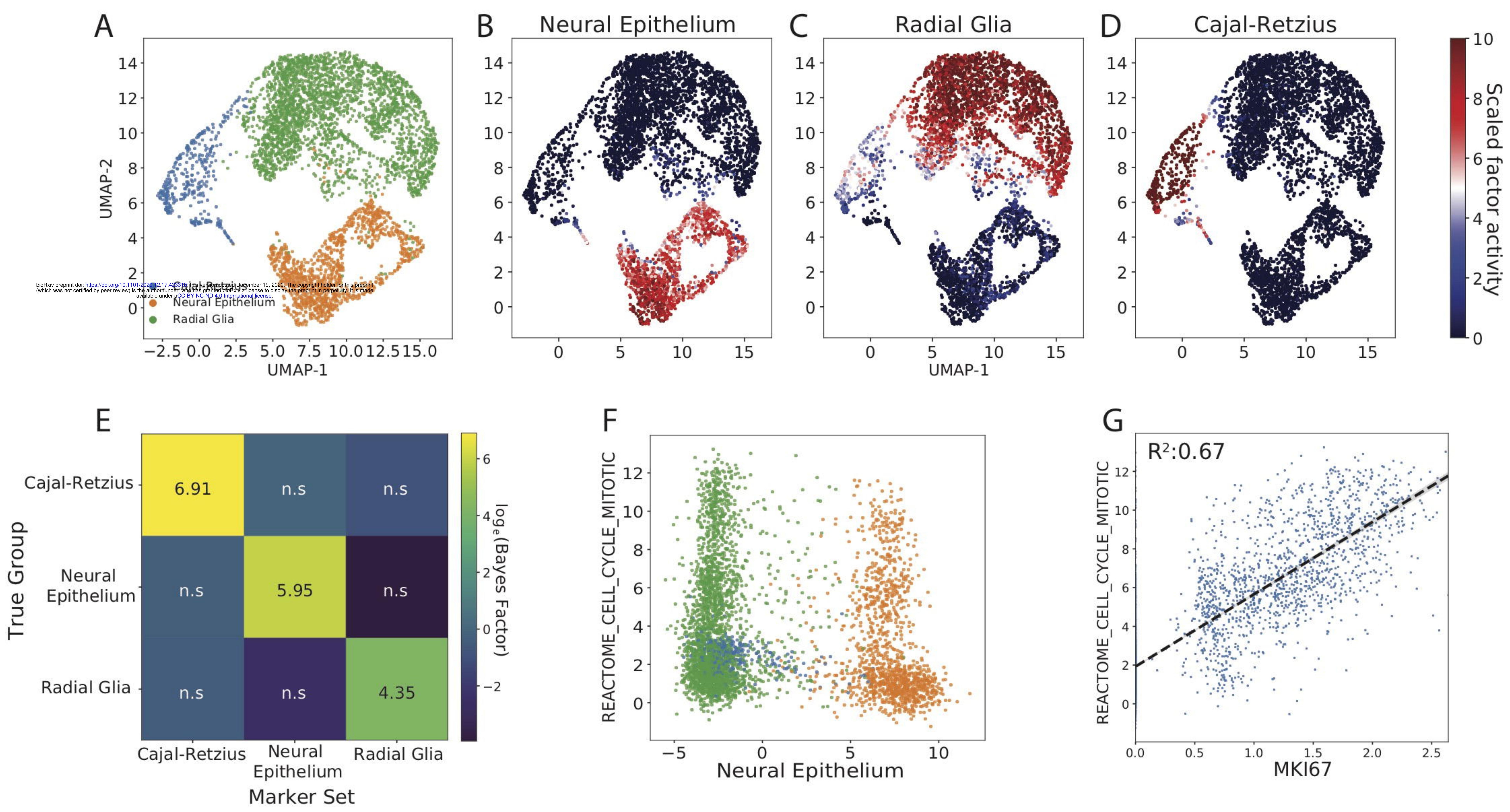
A

B

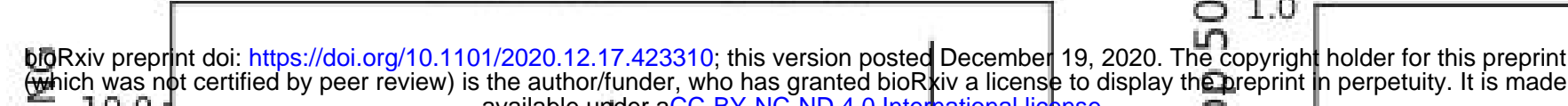
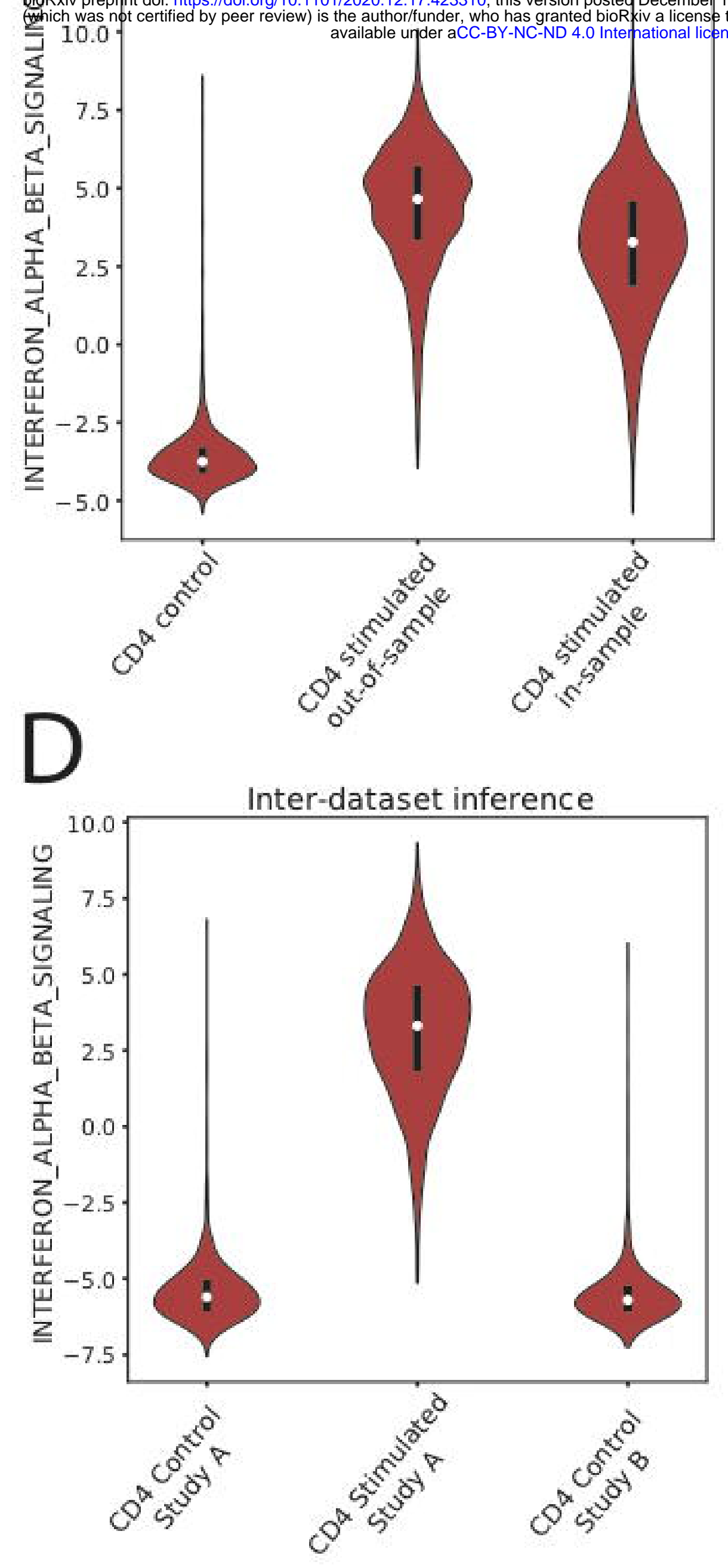

E
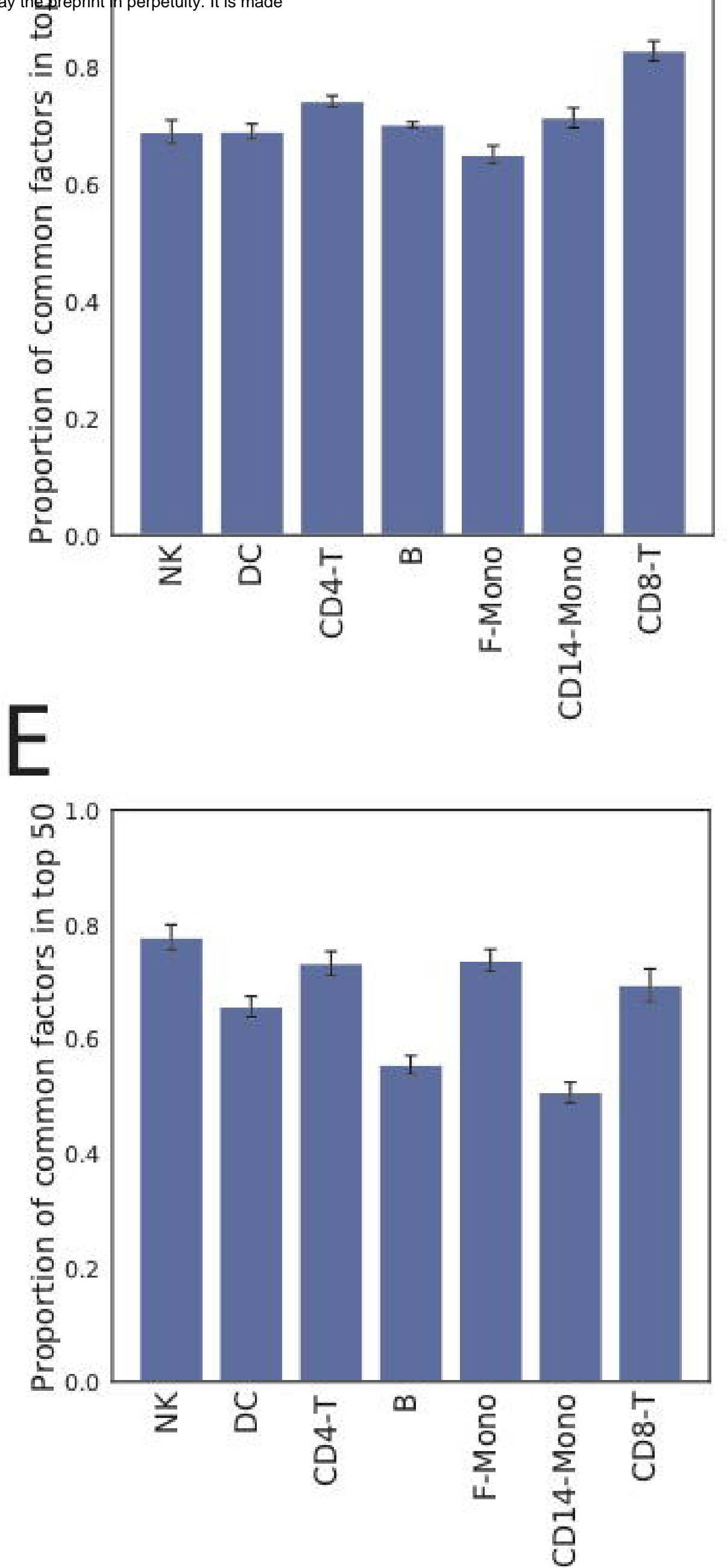

C

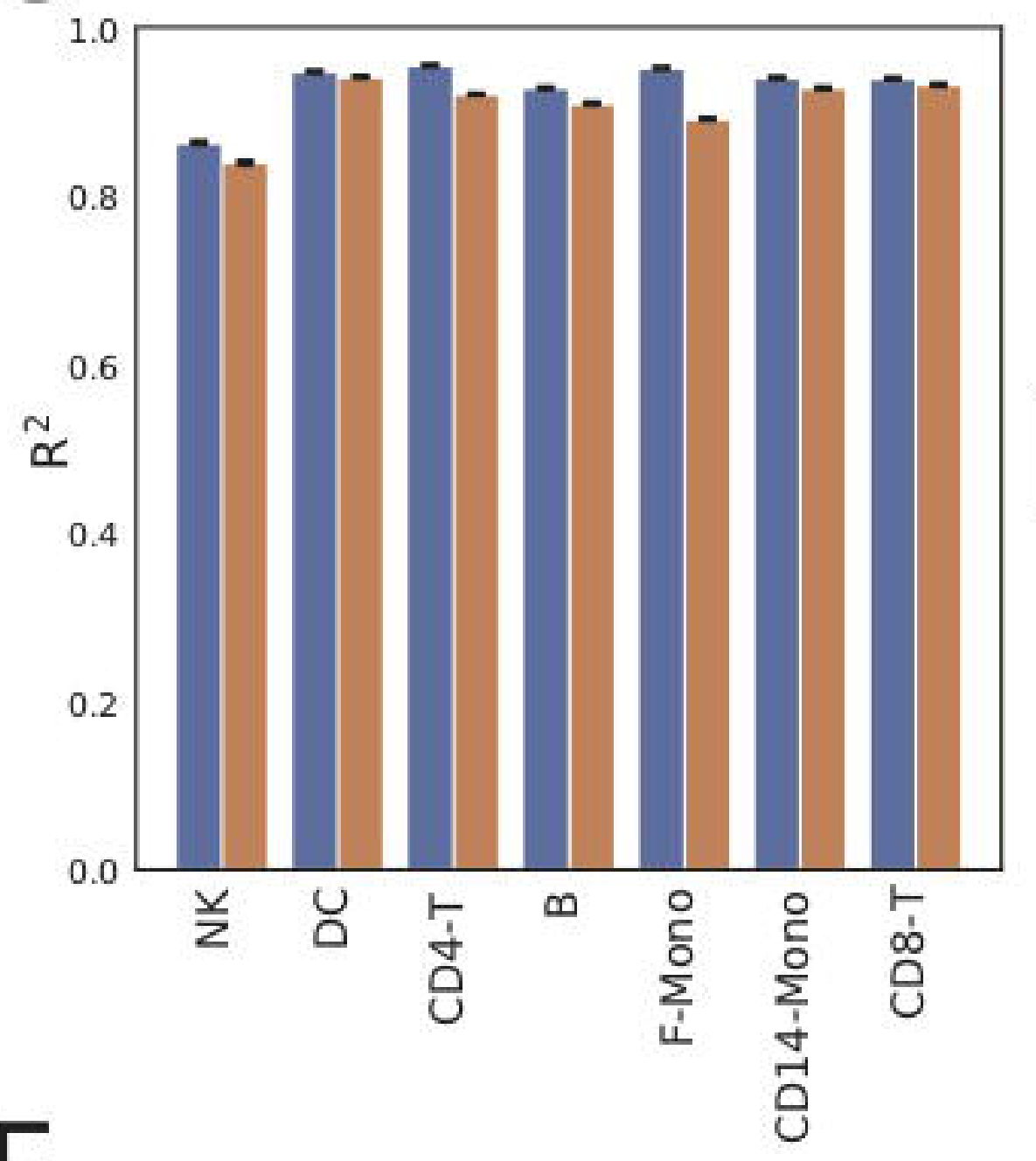

F

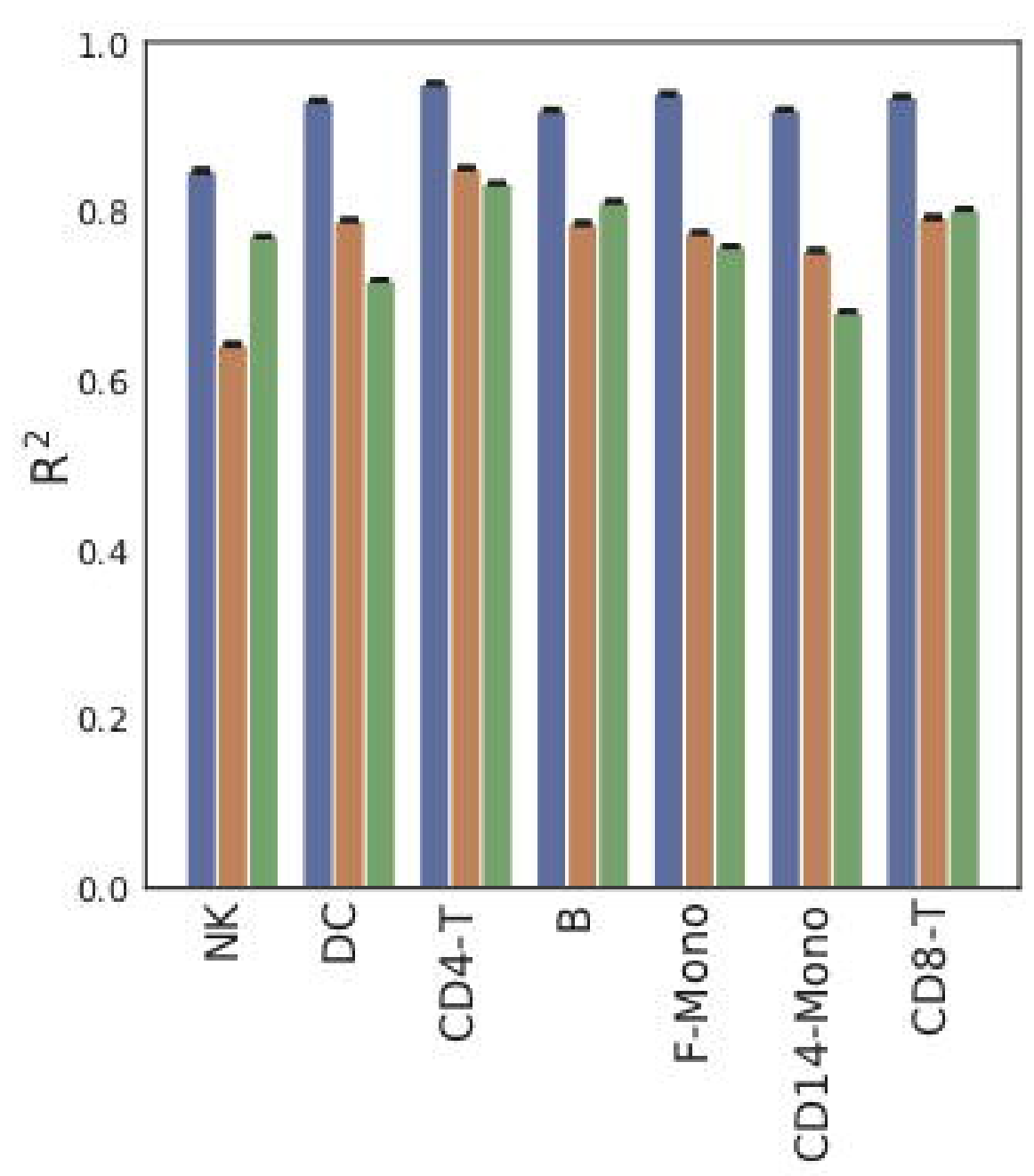

- Study A

- Study B

Study A vs Study B 